\section{Pacientes simulados en la formación de los profesionales de salud: el lado humano de la simulación}

\author{
PHILIPPA MOORE ${ }^{1,2}$, MARÍA INÉS LEIGHTON ${ }^{1,3, a}$, \\ CONSTANZA ALVARADO ${ }^{3, a}$, CECILIA BRALIC ${ }^{3}$
}

\section{Simulated patients in health care training: the human side of simulation}

\begin{abstract}
Simulated patients (SP) are now used in the majority of the institutions that train health care professionals in patient-centered care. This article summarizes the information about the use of SP in health education using information from the literature and from the 15 years' experience in the medical school of the Pontificia Universidad Católica de Chile. It describes the different definitions in use, the roles and scenarios that can be used when teaching with SP and the organization that any institution working with SP should have in order to promote the optimal use of SP. Working with SP allows faculty to center their teaching on their students while keeping the focus on the patient. Students appreciate learning with SP and particularly value feedback from the patients' perspective.
\end{abstract}

(Rev Med Chile 2016; 144: 617-625)

Key words: Education, Professional; Patient Simulation; Teaching.
'Unidad de Pacientes Entrenados, Centro de Educación Médica. Escuela de Medicina (EMUC). Pontificia Universidad Católica de Chile. Santiago, Chile.

2Departamento de Medicina Familiar. Escuela de Medicina. Pontificia Universidad Católica de Chile. Santiago, Chile.

${ }^{3}$ Escuela de Teatro, Facultad de Artes, Pontificia Universidad Católica de

Chile. Santiago, Chile.

${ }^{a}$ Actriz.

Fuente de Apoyo financiero: E equipo de autores recibieron recursos de Proyecto VRI Interdisciplinaria, Pontificia Universidad Católica de Chile 2012-2013. La institución no tuvo influencia en el diseño del estudio; ni en la recolección, análisis o interpretación de los datos; ni en la preparación, revisión o aprobación del manuscrito.

Recibido el 27 de agosto de 2015 , aceptado el 16 de noviembre de 2015.

Correspondencia a: Dra. Philippa Moore Departamento de Medicina Familiar, Pontificia Universidad Católica de Chile. Santiago, Chile. moore@med.puc.cl
E $\mathrm{n}$ la formación de los profesionales de salud en este siglo XXI, se exige una docencia centrada en el estudiante en la cual, además de altos estándares científicos y tecnológicos, se incluyan otros dominios tales como comunicación, profesionalismo, trabajo en equipo, entre otros ${ }^{1}$. El contacto con pacientes siempre ha sido una parte fundamental de la formación de los profesionales de la salud; se entiende que la interacción precoz con pacientes aumenta la motivación de los estudiantes, permite una transición gradual entre los años pre-clínicos y clínicos, y favorece que el estudiante aprenda sobre temas que son difíciles de abordar desde los libros o un plano teórico, tales como empatía, responsabilidad por el paciente, profesionalismo ${ }^{2}$. Por otra parte, la observación de los estudiantes en interacción con sus pacientes permite el aprendizaje basado en competencias y una evaluación en las escalas más altas de la pirámide de Miller³.
El ejercicio de los profesionales de salud ha cambiado dramáticamente en los últimos años ${ }^{4}$. El que la estadía de los pacientes en los hospitales sea cada vez más breve, y que la mayoría de los pacientes hospitalizados tengan una condición de salud grave, hace inadecuado que sean el centro de docencia del gran número de estudiantes que hoy aprendan en los campos clínicos. Las universidades deben asegurar que tienen protocolos claros sobre los temas éticos y legales implicados en la relación paciente-estudiante y contar con un sistema que protege a los pacientes que están involucrados en cualquier aspecto de la educación médica ${ }^{5}$. La nueva Ley de Derechos y Deberes de los pacientes recalca la importancia de un balance favorable en la relación equipo docente-paciente, que se fundamenta en la entrega de una atención centrada en los pacientes, promoviendo una cultura de calidad y seguridad en toda la actividad ${ }^{6}$. Los pacientes en Chile tienen una buena disposición a 
colaborar con la docencia, pero al mismo tiempo exigen ser respetados en su derecho a decidirlo ${ }^{7}$. Con el creciente número de estudiantes en los establecimientos de salud, la presencia de ellos y la observación de la atención médica debe ser organizada de tal manera que no perjudique o incomode a los pacientes ${ }^{8,9}$. En el marco de estos antecedentes es posible observar la existencia de una tensión para el docente clínico: es difícil centrarse en el paciente y en el estudiante en la misma instancia. Buscando una alternativa para abordar esta tensión, la simulación clínica tiene como fin representar experiencias reales de pacientes a través de escenarios adecuadamente guiados y controlados: esto permite al docente centrarse en el estudiante pero enfocado en el paciente ${ }^{10}$. Dos vertientes en simulación han emergido en estos años: la simulación tecnológica con maniquíes y máquinas y la simulación humana en lo cual los simuladores son personas que han sido formados para representar un caso clínico en contextos de docencia. El uso de simulación tecnológica ha sido resumido recientemente en esta revista ${ }^{11}$. Este artículo tiene por objetivo el complementar esa revisión con una sinopsis del uso de la simulación humana en la formación de los profesionales de salud desde la literatura y la experiencia en Chile en la Escuela de Medicina de la Pontificia Universidad Católica de Chile (EMUC).

\section{Definiciones y Desarrollo}

Un paciente simulado (PS) es una persona que no padece una enfermedad sino que la simula o actúa para fines docentes, siendo capacitado para aportar a la docencia o evaluación desde el rol de paciente. Barrows desarrolló el primer trabajo con PS con residentes de neurología en los años 60, publicando en 1993 una revisión del tema ${ }^{12}$. Cuando el paciente simulado, además, ha sido capacitado para repetir su caso varias veces con exactitud se habla de paciente estandarizado (PE). En 1975, Harden introduce los Exámenes Clínicos Objetivos y Estandarizados (ECOE/OSCE), metodología en cual los PE son claves ${ }^{13}$. En la literatura anglosajona el concepto standardized patient se ocupa en términos genéricos como equivalente de PS y $\mathrm{PE}$. El concepto de paciente entrenado surge con la finalidad de tener un nombre genérico para las diferentes personas que han recibido capacitación para aportar como pacientes a la formación de los profesionales de salud e incluye los pacientes simulados, pacientes estandarizados y también pacientes reales, quienes consienten en acudir a una sesión docente en la cual el fin no radicará en conseguir un beneficio propio sino con propósitos netamente docentes.

Actualmente la simulación con PS está siendo utilizada en la mayoría de las escuelas de medicina en Estados Unidos de Norteamérica ${ }^{14}$, Canadá ${ }^{15}$ y Europa ${ }^{16}$, y también en la formación de otros profesionales de la salud ${ }^{17}$ como odontología ${ }^{18}$, fonoaudiología ${ }^{19,20}$, nutrición, kinesiología y enfermería ${ }^{21}$. En Chile, el trabajo con PS comienza en la Escuela de Medicina de la Pontificia Universidad Católica de Chile (EMUC) en 1995 en el programa de Medicina Familiar. En 2000 se introducen los ECOE/OSCE, incluyendo estaciones con pacientes simulados ${ }^{22,23}$. Desde entonces a la fecha el aporte de los PS/PE ha tendido a un crecimiento exponencial en la educación de los profesionales de salud en Chile ${ }^{24}$.

\section{Diversos roles y escenarios en el trabajo con pacientes simulados}

Existe un amplio abanico de diferentes metodologías empleadas con PS, tanto en docencia como en evaluación ${ }^{25}$ (Tabla 1).

\section{Role-play en docencia clínica}

La docencia con PS se puede desarrollar aplicando la metodología de role-play, en la cual un estudiante asume el rol del profesional de salud y entrevista y/o examina al PS como si fuera su paciente. Este último es capaz de interactuar con el "profesional de salud" desarrollando respuestas acordes a los estímulos entregados durante el desarrollo de la entrevista. El rol del docente, capacitado como facilitador, es fundamental para optimizar el aprendizaje con $\mathrm{PS}^{26,27}$. El role-play expone al estudiante a la observación y feedback de sus pares y docentes, y la tensión que agrega una persona externa (el PS) al grupo de aprendices, puede aumentar esta sensación de exposición ${ }^{28}$. Si el docente logra crear un ambiente seguro y ayuda a sus estudiantes a "entrar en el juego", esta tensión puede devenir en un espacio de aprendizaje más complejo y profundo ${ }^{29}$. Un ambiente docente que representa lo más fielmente posible el ambiente 
Tabla 1. Diversos roles y escenarios en el trabajo con pacientes simulados (PS)

\section{Docencia}

- Role-Play en grupos pequeños (con o sin espejo uni-direccional)

o Docente como entrevistador-modelo

o Estudiante como entrevistador

- Entrevista 1 a 1

- Entrevista grupal-varios estudiantes toman en turno entrevistar el paciente

- "Teatro" en aula

- Representación de escenas del mundo médico

o Representación de una entrevista

- Filmación

o Entrevistas 1 a 1 con pacientes simulados

o Pacientes simulados hablando directamente a la cámara

- "Cita" con PS

o Estudiante pido "hora" con PS, le entrevista y PS le da feedback (con o sin observación docente)

\section{Evaluación}

- Osce/Ecoe: estaciones de anamnesis, examen físico, plan de manejo, estaciones híbridos con fantomas y PS

- Long-Case: entrevista completa con PS

- Pacientes Incógnitos: en su policlínico habitual, el estudiante o medico sabe que uno de sus pacientes puede ser un PS, pero no sabe cuál.

- Prueba escrita (respuestas cortas o selección múltiples) con análisis de filmación de PS

en el cual se atiende pacientes reales (ej.: PS con bata y en camilla si el caso es hospitalario) facilita "entrar en el juego". Los pasos para realizar docencia utilizando role-play con PS están resumidos en Tabla 2.

En el trabajo que hemos desarrollado en la EMUC, una de las preguntas comunes que hemos debido abordar es cuándo es el momento adecuado de informar al estudiante que atenderá a un paciente simulado y no a un paciente 'real'. ¿Debería el docente presentar al PS como paciente, como actor o no decir nada? Nuestra experiencia, tal como lo sugiere Kurtz, nos ha demostrado que es preferible que los estudiantes sepan que están trabajando con un PS, para que puedan aprovechar la simulación y optimizar su aprendizaje, atreviéndose a "practicar" más allá de lo que se podría en una situación real, además de no sentirse defraudados en el momento de "la verdad"26.

Los beneficios de incluir PS en la formación de los profesionales de salud son diversos (Tabla 3 ). Los PS son capaces de representar los detalles de un caso en forma fidedigna y mantener uniformidad en su estilo emocional y comunicacional ${ }^{30}$ especialmente si su entrenamiento incluye el uso de vídeo ${ }^{31}$ y si hay una evaluación de su desempeño
Tabla 2. Los pasos para realizar docencia utilizando role-play con pacientes simulados

- Establecer un ambiente seguro

- Explicitar las reglas del grupo

- Asignar las tareas de observación a los estudiantes

- Explorar el auto-conocimiento del estudiante quien toma el rol de entrevistador

- Determinar la duración del role-play

- Facilitar la reflexión inicial del entrevistador

- Facilitar el feedback de los estudiantes observadores

- Reconocer los temas generativas y el "precipicio de aprendizaje" del grupo

- Integrar conocimiento con práctica

- Facilitar el entrevistador en su plan para mejorar

- Resumir lo aprendido

usando instrumentos validados ${ }^{32}$. La interacción "profesional de salud" - PS puede acercarse a representar una entrevista real de tal manera que no hay diferencias en los aprendizajes de competencias cuando se compara PS y pacientes reales en 
Tabla 3. Los beneficios y dificultades del uso de PS en la educación de los profesionales de salud

Beneficios para los estudiantes:

- Menos amenazante que pacientes reales

- Importante para prepararlos para el mundo real

- Aprendizaje activo

- Feedback inmediato

- Practicar situaciones difíciles

- Protección de los pacientes reales

Beneficios para los docentes:

- Disponible cuando, donde se requiere

- Controlado, planificado

- Flexibles

- Experiencias equivalentes

- Puede evaluar objetivos en forma consistente

Beneficios para la institución:

- Enfatiza el enfoque centrada en el paciente

- Evita tratamientos equivocados a los pacientes reales

- Se puede usar en todos los cursos, todos los años

- Costo-efectivo: Permite observación directa Reduce el tiempo del docente

Dificultades en el uso de pacientes simulados

- Nunca un PE es tan real como un paciente real

- No puede representar muchas de los signos físicos

- Ser observado con una persona ajena al grupo (el PS) puede ser estresante

- Tanto los estudiantes como los docentes tienen que "entrar en el juego"

- Requiere tiempo del docente en la preparación del caso y en el piloto

- Requiere una organización especial-entrenador de PE, banco de casos, coordinación curricular

la docencia de pre-grado ${ }^{33-36}$. Sin embargo, hay un llamado a "cuidar la brecha" entre la experiencia simulada y la no-simulada (con pacientes reales), especialmente en el aprendizaje de empatía, profesionalismo y en el caso del trabajo con pacientes con enfermedades psiquiátricas ${ }^{37-39}$. Se recomienda usar casos "simples" en los primeros encuentros de los estudiantes con PS, y luego incluir situaciones que sea difícil que los estudiantes las enfrenten en el mundo real: situaciones de emergencia o de poca frecuencia; entregar una mala noticia o discutir temas delicados como disfunción sexual; entregar un plan de manejo en común acuerdo con el paciente; ayudar a un paciente a un cambio en su estilo de vida, entre otras ${ }^{40}$. En docencia de postgrado el aprendizaje es mayor si los casos son basados en casos desafiantes identificados de la propia experiencia de los residentes. También el trabajo con escenas de simulación tecnológica con maniquíes que incluyen además un PS, simulación híbrida, permiten observar múltiples competencias en una sola instancia.

Los estudiantes valoran la posibilidad de aprender en un clima seguro, donde el error no tiene mayores consecuencias que la posibilidad de generar una instancia para revisar dónde estuvo el error, qué síntoma se pasó por alto o qué pregunta pertinente olvidó de realizar ${ }^{33}$. Los estudiantes perciben que tanto PS como pacientes reales son un gran aporte a su aprendizaje distinguiendo ventajas y desventajas específicas a cada encuentro: un PS nunca puede ser tan real como un paciente real a quien le valoran por su autenticidad, pero la disponibilidad y selección de los pacientes reales puede ser difícil. En cambio, los estudiantes valoran el aprendizaje con PS por el feedback del PS y la posibilidad de practicar sus habilidades comunicacionales ${ }^{41}$. Otra forma de role-play es cuando los propios estudiantes representan a un paciente $\mathrm{y}$ al profesional de salud. Esto tiene el beneficio de disminuir costos, y permite a los estudiantes experimentar la vivencia desde la perspectiva de la persona que padece una determinada patología, lo cual es claramente un beneficio en relación al desarrollo de habilidades empáticas ${ }^{42}$. Sin embargo, algunos estudiantes presentan dificultades al momento de entrevistar en forma natural a un par que encarna el rol de un paciente y aun más complejo es dar un feedback en forma objetiva y desde la perspectiva del paciente ${ }^{43,44}$. El PS está entrenado específicamente para representar el caso en forma fiel y permitir que el role-play fluya en respuesta a las competencias del estudiante. No se ha logrado demostrar mayor aprendizaje con role-play o sin role-play con $\mathrm{PS}^{45}$.

\section{Rol como agente educativo: feedback, PS-docente, estudiante simulado}

A pesar de que el rol principal del PS sea representar un paciente, el aporte que estos brindan a la docencia puede ser tanto mayor. Hay experiencias con estudiantes simulados (para la capacitación de los docentes), administradores simulados (para capacitación en acreditación) y PS-Docente, standarized patient trainer, (cuando está capacitado en enseñar, por ejemplo, el examen físico a los estudiantes sin requerir de la presencia de un académico ${ }^{46}$. El feedback del PS es la acción 
educativa mas valorada por los estudiantes. Un PS bien capacitado es capaz de observar cuidadosamente lo ocurrido durante la entrevista, recordar los detalles de las competencias demostradas por el estudiante y luego entregarle esta información en forma constructiva y acorde con las necesidades de aprendizaje ${ }^{47}$. Este feedback, que puede ser dado en forma verbal, o escrita (usando una pauta de cotejo) puede ser:

- Desde la perspectiva del paciente.

- Sobre las habilidades demostradas por el estudiante durante la entrevista: ej.: si obtuvo toda la información pertinente, si llevó a cabo el examen físico en forma correcta.

En su feedback el PS puede lograr sintetizar una visión objetiva del desempeño del estudiante durante la entrevista con una visión subjetiva desde su propia experiencia como paciente y así complementar el feedback dado por el docente ${ }^{48}$. Hay escasa información sobre la mejor forma de entrenar el PS en feedback ${ }^{41,49}$.

\section{Rol en evaluación}

El uso de PE en estaciones de ECOE/OSCE forma parte del "gold standard" para la evaluación de competencias ${ }^{50}$. En un tiempo determinado, (entre 5 y 30 min según los objetivos del estación), el estudiante debe entrevistar y/o examinar al PE como si fuera su paciente. Un docente-evaluador y/o el PE recogen el desempeño observado en una pauta de cotejo.

Los PE logran repetir el mismo escenario varias veces de manera estándar, sin perder la espontaneidad requerida para poder responder en forma adecuada a las preguntas de cada estudiante ${ }^{51}$. Comparando PE y pacientes reales en un ECOE/ OSCE, se demostró que PE responden en forma más consistente, permite duplicar la estación para permitir múltiples estaciones idénticas y mantienen su estandarización en diferentes centros ${ }^{19,52}$. Los estudiantes se quejan de la sensación de artificialidad en los encuentros con PE en ECOE/OSCE; sin embargo, se plantea que esto se debe más al escenario evaluativo que a la falta de realismo del PE, dado que si el PE consulta en forma incógnita, ni médicos con experiencia distinguen entre un $\mathrm{PE}$ de un paciente real, (tasas de detección 10-20\%) ${ }^{48}$.

Un PE bien capacitado puede completar una pauta de cotejo en forma válida con una concordancia promedio con un equipo de do- cente-evaluadores de $88-92 \%{ }^{53-55}$ y la precisión global de los PE fue de $91 \%$ usando "examinados estandarizados" ${ }^{56,57}$. Si un PE comete un error en su pauta de cotejo tiende a dar crédito al evaluado por algo que no demostró ${ }^{58}$. Desde el 2005, en el OSCE de Stage 2 USMLE (el examen médico nacional de Estados Unidos de Norteamérica) no hay académicos presentes. Los PE están entrenados en completar pautas de cotejo del desempeño del examinado en habilidades comunicacionales y en examen físico. El docente evalúa al estudiante utilizando la información recogida por el PE y la información escrita por el examinado en la ficha clínica $^{59,60}$. Con un control de calidad estricta, los PE ofrecen una forma costo-efectiva para organizar estos evaluaciones que son de alto riesgo (“high-stakes") para los evaluados ${ }^{61}$.

\section{Organización de pacientes simulados}

Utilizando ejemplos de nuestra experiencia en EMUC, describimos las especificaciones propuesta por Wallace para el óptimo trabajo con $\mathrm{PS}^{47}$.

\section{El presupuesto}

Introducir simulación en el currículo tiene un costo importante. Hay esfuerzo en estimar el costo-efectividad de simulación; no es una tarea fácil, requiere describir en términos monetarios el valor de la seguridad para el paciente y su calidad de vida $^{62}$. Al contrario a la simulación con fantomas que puede requerir gran inversión al inicio y luego un mínimo costo de mantención, un programa de PS requiere un presupuesto anual para financiar su desempeño en la docencia y/o evaluación, además de un costo fijo para la organización, contratación y capacitación de las personas que se desempeñan como PS. El uso de PS debe ser programado en el currículo en forma lógica y de tal manera que optimice su aporte a la formación de profesionales de salud. Medidas como buscar un PS de menor costo o minimizar su capacitación no son efectivas; cuando no hay presupuesto, puede ser mejor buscar otros métodos alternativos ${ }^{63}$.

\section{Contratación de staff del programa PS}

El rol de quien dirige y del "coach" o coordinador de PS son fundamentales para el óptimo uso de $\mathrm{PS}^{64}$. Sus roles están resumidos en Tabla 4. En nuestra experiencia en EMUC, el coach debe ser una persona con formación en teatro y con 
Tabla 4. Responsabilidades de staff en un Programa PS

\begin{tabular}{|ll|}
\hline Responsabilidad de Director Académico & Responsabilidad de Coach/Coordinador \\
\hline Políticas de Reclutamiento & Reclutamiento \\
\hline Evaluación del Programa PS & Desarrollo y mantención de base de datos \\
\hline Control de calidad de PS & Coordinación con docentes re- casos, guiones, fechas \\
\hline Capacitación PS en temas de educación: ej. feedback & Capacitación PS en sus casos \\
\hline Fidelización de PS & Bienestar de los PS \\
\hline
\end{tabular}

experiencia como PS, además de sus capacidades en coaching. Para aproximadamente $1.880 \mathrm{~h}$ anuales de representaciones PS se requiere un "coach" contratado por un mínimo de $33 \mathrm{~h}$ semanales.

\section{Creación del caso}

La planificación según los objetivos de la docencia de los casos que los PS deben representar es un trabajo en conjunto entre el docente y el "coach" PS. El docente requiere tiempo protegido para escribir los objetivos y la patología del paciente, el "coach" enriquece el guión entregado por el docente, creando un personaje y sugiriendo metodologías, lo cual se chequea con el docente en el piloto (Tabla 5). Si la metodología propuesta requiere feedback escrito, es necesaria diseñar pautas de cotejo para el docente y/o paciente simulado. Cuando el PS conoce las pautas de cotejo de antemano, facilita que no entregue de forma gratuita información que debe ser indagada en forma especifica por el estudiante.

\section{La selección cuidadosa de personas}

Se busca las personas que cumplen con la edad y físico requerido para el caso. Se puede reclutar con un aviso en el diario, en el cual se hace un llamado a personas que reúnan un determinado número de características como sexo, edad, contextura, nivel educacional, entre otras. En EMUC (y otras escuelas de medicina $)^{65}$ reclutamos principalmente (aunque no exclusivamente) a actores profesionales. Es nuestra experiencia que el actor profesional tiene competencias que le permiten asumir el rol de PS con mayor facilidad que personas que no tiene formación en teatro (Tabla 6). Sin embargo, no todos los actores son buenos PS. El actor debe ser capaz de dejar de lado su protagonismo y reconocer las necesidades que el escenario pedagógico clínico presenta. Aquí lo central no es su actuación o desempeño como paciente, sino el

\section{Tabla 5. Elementos fundamentales en un guión PS}

El guión debe incluir

- Los objetivos (¿qué se espera que aprendan los estudiantes con este caso?)

- La metodología docente (¿se representará toda la entrevista/solamente una parte?, ¿en forma continua/con interrupciones?, ¿cuántas veces debe repetir el caso?, ¿cuándo debe dar feedback?, ¿cuándo puede salirse del rol?)

- Patología del paciente-anamnesis completo

- Perspectiva del paciente de su enfermedad: miedos, preocupaciones, emociones

- Personalidad del paciente

- Información social

Tabla 6. Características de actores profesionales que facilita su rol como PS

- Experiencia en aprender guiones y representar personajes

- Sin inhibición de ser observado

- Entrenado en técnicas* que le permiten conectar con sus emociones de manera rápida y efectiva como el momento lo requiera. Ej: Aunque nunca haya sido diagnosticado de cáncer, es capaz de "sentir" la angustia y desesperación que esto puede significar

- Experiencia desde el cine a repetir con gran precisión la gestualidad, el tono de voz, la secuencia emocional y la veracidad con que desarrolla un rol

- Capacitado para "limpiarse" de la emoción con rapidez que permite empezar en neutralidad la siguiente entrevista protegerse del daño a largo plazo que se podría producirse al representar sufrimiento y enfermedades graves

- Capacidad de desdoblamiento que les permite observarse a sí mismos durante una instancia de actuación. Ej: el actor cuando está participando en una entrevista clínica como PS, es capaz de verse desde el exterior, como si fuera una pequeña cámara que está registrando todo lo que allí está sucediendo, sin perder la concentración de la entrevista misma

*Stanislavsky C. Un actor se prepara. Trigésimo Edición; México, Editorial Diana, 1999. 
contexto de aprendizaje en juego y, por lo tanto, su relación con el estudiante y docente.

\section{Entrenamiento en simulación y estandarización}

El entrenamiento del PS es de 4 a $12 \mathrm{~h}$, dependiendo de la complejidad del caso. Debe incluir un piloto con el docente quien creó el caso.

\section{Control de calidad y evaluación de la docencia}

La evaluación intermitente junto con feedback sobre su desempeño son factores importante en la mantención de la calidad de desempeño de los $\mathrm{PS}^{61}$. En EMUC realizamos visitas "inesperadas" para observar la docencia, y recogemos periódicamente las opiniones de los docentes y los estudiantes sobre el desempeño de nuestros pacientes simulados. En cada estación de ECOE/OSCE los evaluadores tienen la posibilidad de expresar su opinión sobre el desempeño de su PE; en ECOE/ OSCE de alto riesgo ("high-stakes"), para los evaluados, se filma las estaciones creando un registro que permite evaluar la estandarización y correcta representación de los casos.

\section{Mantener el entusiasmo y un sentido de pertenencia a un gran proyecto de formar profesionales de salud de excelencia}

Un programa PS es incompleto si no crea instancias para fortalecer la misión de los PS de aportar a la formación de profesionales de salud de excelencia: tales como capacitación en temas relacionado con educación (ej. feedback, como afinar su observación), feedback sobre su desempeño. Además, es importante cuidar la salud de los PS, por ejemplo, procurar tener una colación cuando tiene que trabajar largas horas.

\section{Conclusiones}

La simulación con PS es una herramienta eficaz para la formación de profesionales de la salud, ya que facilita a los estudiantes una visión holística del paciente. Los PS no surgen con la pretensión de reemplazar a los pacientes reales ni a los docentes, sino a fin de colaborar con la docencia médica a través de un espacio de experimentación que, partiendo del error como punto de partida, y utilizando la potencia del aprendizaje experimental, espera colaborar con el correcto desempeño de los estudiantes y profesionales de salud. La conexión que brindan los PS entre medicina y teatro abre posibilidades de un trabajo interdisciplinario que tiene el potencial de enriquecer la educación de los profesionales de salud con herramientas del mundo teatral. Siendo una herramienta con un costo importante, los PS deben ser utilizados en forma óptima permitiendo una docencia centrada en el estudiante cuidando la seguridad del paciente.

\section{Referencias}

1. The CanMeds Framework [Internet]. Available from: http://www.royalcollege.ca/portal/page/portal/rc/canmeds/framework.

2. Dornan T, Littlewood S, Margolis SA, Scherpbier A, Spencer J, Ypinazar V. How can experience in clinical and community settings contribute to early medical education? A BEME systematic review. Med Teach 2006; 28 (1): 3-18.

3. Shumway JM, Harden RM. AMEE Guide No 25: The assessment of learning outcomes for the competent and reflective physician. Med Teach 2003; 25 (6): 569-84.

4. Jiménez JP. [Doctors' strategies to cope with the crisis in medical profession]. Rev Med Chile 2005; 133 (6): 707-12.

5. Sayer M, Bowman D, Evans D, Wessier A, Wood D, Brook R. Use of patients in professional medical examinations: current UK practice and the ethicolegal implications for medical education. BMJ 2002; 324 (February): 404-7.

6. Ministerio de Salud Chile. LEY-20.584 Derechos y Deberes de las Personas en Atención de Salud [Internet]. Biblioteca del Congreso Nacional. 2012. Disponible de: http://www.leychile.cl/Navegar?idNorma=1039348.

7. Beca JP, Browne F, Valdebenito C, Bataszew A, Martínez MJ. Relación estudiante-enfermo: Visión del paciente. Rev Med Chile 2006; 134: 955-9.

8. Jagsi R, Lehmann LS. The ethics of medical education. BMJ. 2004; 329 (7461): 332-4.

9. Grupo de Estudios de Ética Clínica Sociedad Médica de Santiago. Dimensión ética en la organización de la atención de salud. Rev Med Chile 2013; 141: 780-6.

10. Nestel D, Tabak D, Tierney T, Layat-Burn C, Robb A, Clark S, et al. Key challenges in simulated patient programs: an international comparative case study. BMC Med Educ. 2011; 11: 69.

11. Corvetto M, Bravo MP, Montaña R, Utili F, Escudero E, Boza C, et al. [Simulation in medical education: a synopsis]. Rev Med Chile 2013; 141 (1): 70-9.

12. Barrows HS. An Overview of the Uses of Standardized 
Patients for Teaching and Evaluating Clinical Skills. Acad Med 1993; 68 (6): 443-51.

13. Harden RM, Gleeson FA. Assessment of clinical competence using an objective structured clinical examination (OSCE). Med Educ 1979; 13 (1): 41-54.

14. Huang GC, Sacks H, Devita M, Reynolds R, Gammon $\mathrm{W}$, Saleh M, et al. Characteristics of simulation activities at North American medical schools and teaching hospitals: an AAMC-SSH-ASPE-AACN collaboration. Simul Healthc 2012; 7 (6): 329-33.

15. Howley LD, Gliva-McConvey G, Thornton J. Standardized patient practices: initial report on the survey of US and Canadian medical schools. Med Educ Online 2009; 14: 7.

16. Cantillon P, Stewart B, Haeck K, Bills J, Ker J, Rethans J-J. Simulated patient programmes in Europe: collegiality or separate development? Med Teach 2010; 32 (3): e106-10.

17. May W, Park JH, Lee JP. A ten-year review of the literature on the use of standardized patients in teaching and learning: 1996-2005. Med Teach 2009; 31 (6): 487-92.

18. Carey JA, Madill A, Manogue M. Communications skills in dental education: a systematic research review. Eur J Dent Educ 2010; 14 (2): 69-78.

19. Hill AE, Davidson BJ, Theodoros DG. The performance of standardized patients in portraying clinical scenarios in speech-language therapy. Int J Lang Commun Disord. Jan; 48 (6): 613-24.

20. Dinsmore BF, Bohnert C, Preminger JE. Standardized patients in audiology: a proposal for a new method of evaluating clinical competence. J Am Acad Audiol 2013; 24 (5): 372-92.

21. Shin S, Park J-H, Kim J-H. Effectiveness of patient simulation in nursing education: Meta-analysis. Nurse Educ Today 2015; 35 (1): 176-82.

22. Moraga L, Moore P. [Helping fish to live? Metamorphosis of a structured objective clinical evaluation (ECOE) for a family medicine intern in the last year of medical school]. Aten Primaria 2010; 42 (5): 302-3.

23. Triviño X, Vásquez A, Mena A, López A, Aldunate M, Varas M, et al. [Application of Objective Structured Clinical Examination (OSCE) for pediatric internship assessment in two schools of medicine]. Rev Med Chile 2002; 130 (7): 817-24.

24. Solís I, Bozzo S, Kunakov N. Pacientes estandarizados en la formación de habilidades clínicas, en educación médica de pregrado. Rev Med Chile 2013; 141 (9): 12167.

25. Rethans J-J, Grosfeld FJM, Aper L, Reniers J, Westen $\mathrm{JH}$, van Wijngaarden JJ, et al. Six formats in simulated and standardized patients use, based on experiences of
13 undergraduate medical curricula in Belgium and the Netherlands. Med Teach 2012; 34 (9): 710-6.

26. Kurtz S, Silverman J, Draper J. Teaching and Learning Communication Skills in Medicine. Radcliffe Publishing Ltd; 2004.

27. Bylund CL, Brown RF, di Ciccone BL, Levin TT, Gueguen JA, Hill C, et al. Training faculty to facilitate communication skills training: Development and evaluation of a workshop. Patient Educ Couns 2008; 70 (3): 430-6.

28. Stobbs N. Role-play without humiliation: is it possible? Clin Teach 2015; 12 (2): 128-30.

29. Vella J. Learning to Listen, Learning to Teach: The Power of Dialogue in Educating Adults. Revised ed. San Francisco: Jossey-Bass; 2008.

30. Erby L H, Roter DL, Biesecker BB. Examination of standardized patient performance: accuracy and consistency of six standardized patients over time. Patient Educ Couns 2011; 85 (2): 194-200.

31. Schlegel C, Bonvin R, Rethans JJ, van der Vleuten C. The use of video in standardized patient training to improve portrayal accuracy: A randomized post-test control group study. Med Teach 2014; 14: 1-8.

32. Wind LA, Dalen J Van, Muijtjens AMM, Rethans J. Assessing simulated patients in an educational setting: the MaSP (Maastricht Assessment of Simulated Patients). Med Educ 2004; 38: 39-44.

33. Bokken L, Rethans J-J, Jöbsis Q, Duvivier R, Scherpbier $A$, van der Vleuten C. Instructiveness of real patients and simulated patients in undergraduate medical education: a randomized experiment. Acad Med 2010; 85 (1): 148-54.

34. Gilliland WR, Pangaro LN, Downing S, Hawkins RE, Omori DM, Marks ES, et al. Standardized Versus Real Hospitalized Patients to Teach History-Taking and Physical Examination Skills. Teach Learn Med 2006; 18 (3): 188-95.

35. Helfer RE, Black MA, Teitelbaum H. A comparison of pediatric interviewing skills using real and simulated mothers. Pediatrics 1975; 55 (3): 397-400.

36. Hudson J. Teaching clinical skills with patient resources. Can Fam physician 2014; 60: 674-7.

37. Yardley S, Irvine AW, Lefroy J. Minding the gap between communication skills simulation and authentic experience. Med Educ 2013; 47 (5): 495-510.

38. Brenner AM. Uses and limitations of simulated patients in psychiatric education. Acad psychiatry 2009; 33 (2): 112-9.

39. Krahn LE, Sutor B, Bostwick JM. Conveying emotional realism: a challenge to using standardized patients. Acad Med 2001; 76 (3): 216-7.

40. Kneebone R, Nestel D, Wetzel C, Black S, Jacklin R, 
Aggarwal R, et al. The human face of simulation: patient-focused simulation training. Acad Med 2006; 81 (10): 919-24.

41. Bokken L, Linssen T, Scherpbier A, van der Vleuten C, Rethans J-J. Feedback by simulated patients in undergraduate medical education: a systematic review of the literature. Med Educ 2009; 43 (3): 202-10.

42. Lane C, Rollnick S. The use of simulated patients and role-play in communication skills training: a review of the literature to August 2005. Patient Educ Couns 2007; 67 (1-2): 13-20.

43. Rollnick S, Kinnersley P, Butler C. Context-bound communication skills training: development of a new method. Med Educ 2002; 36 (4): 377-83.

44. Nestel D, Tierney T. Role-play for medical students learning about communication: guidelines for maximising benefits. BMC Med Educ 2007; 7: 3.

45. Koponen J, Pyörälä E, Isotalus P. Comparing three experiential learning methods and their effect on medical students' attitudes to learning communication skills. Med Teach 2012; 34 (3): e198-207.

46. Nestel D, Clark S, Tabak D, Ashwell V, Muir E, Paraskevas $\mathrm{P}$, et al. Defining responsibilities of simulated patients in medical education. Simul Healthc 2010; 5 (3): 161-8.

47. Wallace P. Coaching Standardized Patients: For Use in the Assessment of Clinical Competence. First. New York: Springer Publishing Company; 2007.

48. Williams RG. Have Standardized Patient Examinations Stood the Test of Time and Experience? Teach Learn Med 2004; 16 (2): 215-22.

49. Hatchett P, Haun C, Goldenhaur L. Training Standardized Patients to Give Feedback to Medical Trainees: The State of the Art. University of Cincinnati College of Medicine; 2004. Disponible en http://www.aspeducators.org/files/project_awards/1280872305.pdf

50. Carraccio C, Englander R. The objective structured clinical examination: a step in the direction of competency-based evaluation. Arch Pediatr Adolesc Med 2000; 154 (7): 736-41.

51. Adamo G. Simulated and standardized patients in OSCEs: achievements and challenges 1992-2003. Med Teach 2003; 25 (3): 262-70.

52. Tamblyn RM, Klass DJ, Schnabl GK, Kopelow ML. The accuracy of standardized patient presentation. Med Educ 1991; 25 (2): 100-9.

53. Heine N, Garman K, Wallace P, Bartos R, Richards A. An analysis of standardised patient checklist errors and their effect on student scores. Med Educ 2003; 37 (2): 99-104.

54. Park J, Ko J, Kim S, Yoo H. Faculty observer and standardized patient accuracy in recording examinees' behaviors using checklists in the clinical performance examination. Korean J Med Educ 2009; 21 (3): 287 97.

55. McLaughlin K, Gregor L, Jones A, Coderre S. Can standardized patients replace physicians as OSCE examiners? BMC Med Educ 2006; 6: 12.

56. Worth-Dickstein H, Pangaro LN, MacMillan MK, Klass DJ, Shatzer JH. Use of "Standardized Examinees" to Screen for Standardized-Patient Scoring Bias in a Clinical Skills Examination. Teach Learn Med 2005; 17 (1): 9-13.

57. Pangaro LN, Worth-Dickstein H, Macmillan MK, Klass DJ, Shatzer JH. Performance of "standardized examinees" in a standardized-patient examination of clinical skills. Acad Med 1997; 72 (11): 1008-11.

58. Vu NV, Marcy MM, Colliver JA, Verhulst SJ, Travis TA, Barrows HS. Standardized (simulated) patients' accuracy in recording clinical performance check-list items. Med Educ 1992; 26 (2): 99-104.

59. Whelan GP, Boulet JR, McKinley DW, Norcini JJ, van Zanten M, Hambleton RK, et al. Scoring standardized patient examinations: lessons learned from the development and administration of the ECFMG Clinical Skills Assessment (CSA). Med Teach 2005; 27 (3): 200-6.

60. Boulet JR, Smee SM, Dillon GF, Gimpel JR. The use of standardized patient assessments for certification and licensure decisions. Simul Healthc 2009; 4 (1): 35-42.

61. Furman GE. The role of standardized patient and trainer training in quality assurance for a high-stakes clinical skills examination. Kaohsiung J Med Sci 2008; 24 (12): 651-5.

62. Fletcher JD, Wind AP. Cost Considerations in Using Simulations for Medical Training. Mil Med 2013; 178 (10S): 37-46.

63. Bergus GR, Woodhead JC, Kreiter CD. Trained lay observers can reliably assess medical students' communication skills. Med Educ 2009; 43 (7): 688-94.

64. Ker JS, Dowie A, Dowell J, Dewar G, Dent JA, Ramsay $\mathrm{J}$, et al. Twelve tips for developing and maintaining a simulated patient bank. Med Teach 2005; 27 (1): 4-9.

65. Pascucci RC, Weinstock PH, O'Connor BE, Fancy KM, Meyer EC. Integrating Actors Into a Simulation Program. Simul Healthc J Soc Simul Healthc 2014; 9 (2): 120-6. 